\title{
Infrared Observations of Nova Muscae 1991: Black Hole Mass Determination from Ellipsoidal Variations
}

\author{
Dawn M. Gelino, ${ }^{1,2}$ \\ dleeber@nmsu.edu \\ Thomas E. Harrison, ${ }^{2}$ \\ tharriso@nmsu.edu \\ \& Bernard J. McNamara \\ bmcnamar@nmsu .edu \\ Department of Astronomy, \\ New Mexico State University, Las Cruces, NM 88003
}

\begin{abstract}
We have obtained infrared photometry for the soft x-ray transient GU Mus. We present $J$ and $K_{s}$ band light curves modeled with WD98, the newest version of the Wilson-Devinney light curve modeling code. Using detailed models for the expected ellipsoidal variations due to the non-spherical secondary star, we show that the most likely value for the orbital inclination is $54 \pm 1.5^{\circ}$. This inclination angle is consistent with those previously published, but has a much smaller error. This inclination implies a primary black hole mass of $6.95 \pm 0.6 \mathrm{M}_{\odot}$. While we do not see any evidence for contamination of our infrared light curves from other sources in the system, a conservative model with a contamination level of $15 \%$ increases the uncertainty in the inclination angle to $54_{-1.5}^{o+4}$.
\end{abstract}

Subject headings: binaries: close - stars: black holes - stars: individual (Nova Muscae 1991) - stars: low mass - stars: variables: other

\section{Introduction}

Soft X-Ray Transients (SXTs) are binary systems believed to contain a black hole primary and low mass secondary star. They display large and sudden x-ray and optical outbursts that are

\footnotetext{
${ }^{1}$ formerly Dawn M. Leeber

${ }^{2}$ Visiting Astronomer, Cerro Tololo Inter-American Observatory, National Optical Astronomy Observatory, which is operated by the Association of Universities for Research in Astronomy, Inc. (AURA) under cooperative agreement with the National Science Foundation.
} 
believed to be the result of a sudden, dramatic increase in the mass accretion rate onto the compact object. In quiescence, SXTs are very faint at x-ray and optical wavelengths, however, in this state, the secondary stars can dominate the system luminosity.

GU Mus (=Nova Muscae 1991; XN Mus 91; GRS 1124-68) was discovered by the Ginga and Granat satellites on January 9, 1991 (Makino 1991; Lund 1991). Since then, it has been studied by Remillard et al. (1992), Orosz et al. (1996; hereafter OBMR), Casares et al. (1997; hereafter CMCMR), Shahbaz, Naylor, \& Charles (1997; hereafter SNC), and references therein. These authors have found an orbital period of 10.38 hours, a secondary star spectral type of K4V, and a secondary star radial velocity semi-amplitude of $406 \pm 7 \mathrm{~km} \mathrm{~s}^{-1}$. GU Mus has an implied mass function, the minimum mass of the compact primary object, of $3.01 \pm 0.15 \mathrm{M}_{\odot}(\mathrm{OBMR})$. This mass function combined with the radial velocity curve of the secondary star, established the primary object as a dynamical black hole. Both in outburst and quiescence, GU Mus closely resembles the prototype black hole SXT, V616 Mon (A0620-00).

In quiescence, most SXT's light curves reveal ellipsoidal variations ascribed to the secondary star. Since the secondary star fills its Roche lobe, the surface area seen by an observer on Earth changes as the star orbits the compact object. This changing line-of-sight surface area corresponds to a changing apparent brightness. The amplitude of the ellipsoidal variations is determined by the orbital inclination angle of the system. By combining the orbital inclination angle with the observed mass function, the SXT system parameters can be determined. To this end, we have obtained new infrared observations of GU Mus and model its ellipsoidal light curves using a sophisticated light curve modeling program.

Other authors have also attempted to model observed light curves of GU Mus and other SXTs. In the case of GU Mus all but one of these papers, SNC, have observed the SXT in the optical. If one is searching for the purest ellipsoidal variations, one should observe at a wavelength where the secondary star provides the majority of the system luminosity. In the optical, the accretion disk and hot spot can contaminate, if not dominate, the system luminosity. Even in quiescence, weak and variable accretion might be taking place, and there could be a modest amount of dilution of the optical light curve by the accretion disk, hot spot, and possible heating of the secondary star (SNC). On the other hand, in SXTs with K and M type companions, the secondary star can dominate the quiescent binary's luminosity in the near-infrared. For GU Mus, CMCMR find that the secondary star supplies between $85-88 \%$ of the observed flux in the $R$ band. This percentage will be even higher at longer wavelengths. Therefore, observations in the infrared will reveal more genuine ellipsoidal variations than observations in the optical.

The only way to obtain the orbital inclination angle in non-eclipsing binary systems is from modeling. Previously published inclination angles for GU Mus range from $39^{\circ}$ (lower limit from Antokhina \& Cherepashchuk 1993) all the way to $83^{\circ}$ (upper limit from Hua \& Lingenfelter 1993). More recently, the inclination has been refined to $54_{-15}^{o+20}$ by SNC, and $60_{-6}^{o+5}$ by OBMR. These translate into primary mass ranges of $3.8 \mathrm{M}_{\odot}-10.5 \mathrm{M}_{\odot}$, and $5 \mathrm{M}_{\odot}-7.5 \mathrm{M}_{\odot}$, respectively. 
The rather large ranges in the inclination angles and primary masses that have been published for GU Mus and other SXT systems, has prompted us to determine whether we could more precisely determine the primary mass of the black holes in these SXTs, by using the most recent version of the Wilson-Devinney light curve modeling program to model new infrared observations. We have observed five SXTs in the infrared, but have chosen to present results on GU Mus first out of our sample, since it is the simplest of these systems to model. With no hot spot and very low accretion rate, we probably have the best case scenario for modeling relatively uncontaminated ellipsoidal variations.

In section 2, we describe our observations and data reduction process, as well as present our infrared photometric light curves. Section 3 describes our infrared light curve modeling procedure using WD98, the newest version of the Wilson-Devinney light curve modeling code. We provide details of how we chose the relevant input parameters, present the resulting models at $J$, and $K_{s}$, and discuss the error in the orbital inclination angle. Finally, Section 4 discusses the implications of the models, and compares our results to those previously published.

\section{Observations \& Data Reduction}

GU Mus was observed using OSIRIS (see Pogge et al. 1999) on the Victor M. Blanco 4$\mathrm{m}$ telescope at the Cerro Tololo Inter-American Observatory on 2000 February 20 and 21. On February 20, GU Mus was observed from 1:06 to 9:08 UT with the camera at the f/7 plate scale (0.161" / pixel). On 21 February from 2:16 to 9:12 UT we observed GU Mus with the camera at the $\mathrm{f} / 2.8$ plate scale $(0.403$ " / pixel) due to poorer seeing conditions. With an orbital period of about 10.38 hours, the first observing session covered two-thirds of an orbital period, while the second covered three-quarters of an orbital period. Photometric data were obtained in the OSIRIS $J\left(\lambda_{c}=1.215 \mu \mathrm{m}\right)$ and $K_{s}\left(\lambda_{c}=2.157 \mu \mathrm{m}\right)$ filters. Our observing sequence consisted of a single $J$ image at one position, a beam switch, two additional $J$ images, a beam switch back to the original position, and then one more $J$ image (an ABBA sequence). We then switched to the $K_{s}$ filter and repeated the procedure. Each individual f/7 (f/2.8) $J$ image consisted of 1 frame of 180 (75) seconds, while the corresponding f/7 (f/2.8) $K_{s}$ images consisted of 2 (10) coadded frames of 35 (4) seconds each.

Dome flats at both plate scales were obtained. Before processing, all of the data were linearized using the irlincor package in IRAF with the coefficients supplied in the OSIRIS User's Manual (Pogge et al. 1999). After averaging the two images at one position, we subtracted them from the average of the two images at the other position. These sky, and bias-subtracted images were then

flat fielded using the appropriate dome flat. Figure 1 shows a $J$ band image of GU Mus and the five nearby field stars used for the differential photometry.

Aperture photometry was performed on GU Mus and the five nearby field stars shown in Figure 1. Using the IRAF phot package, a differential light curve in both $J$ and $K_{s}$ was generated 
with each $\mathrm{f} / 7$ point being the average of the two beam switched images, and each $\mathrm{f} / 2.8$ point being the average of four beam switched images. Our differential photometric results show that over the course of our observations, the comparison stars did not vary more than expected from photon statistics. The final $J$ and $K_{s}$ differential light curves of GU Mus, phased to the SNC ephemeris, are presented in Figure 2.

Optical observations of GU Mus were taken with the Cassegrain Focus CCD Imager on the 0.9 meter telescope at CTIO on 2001 March 14 at 3:40 UT. Data were obtained in the $V$, $R$, and $I$ bandpasses for the purpose of determining the quiescent optical colors of the system. The 600 second exposures were zero corrected and flat fielded before aperture photometry was performed. Landolt standard fields were also observed in these optical bands, and were used to correct the apparent magnitudes of GU Mus. The resulting colors can be found in Table 1, along with previously published optical colors for this sytem.

\section{Modeling the Light Curve of GU Mus}

Before we can begin modeling the light curves of GU Mus, we have to determine a large number of parameters. Some of these are intrinsic to the binary system (Sect. 3.1), while others arise from the study of stellar atmospheres (Sects. $3.3 \& 3.4$ ).

\subsection{System Parameters}

The most important input parameter is the nature of the secondary star. In 1996, OBMR compared their observed spectrum of GU Mus to the spectra of various comparison stars, and determined a spectral type of $\mathrm{K} 4 \mathrm{~V} \pm 1$ for the secondary star. In 1997, CMCMR found that the best match to their $\mathrm{H} \alpha$ spectrum was that of a K3V - K4V. Both sets of authors state that the secondary star in GU Mus must be slightly evolved since a 'normal' K4V would underfill its Roche lobe. The secondary can not be a giant, however, since then it would not fit into an orbit with the observed orbital period.

Knowing the secondary's spectral type does not mean that the mass of the secondary is also known. These are most likely not 'normal' stars, as they have survived a supernova explosion and are losing mass. Also as just noted, GU Mus probably has a slightly evolved secondary. Determining the mass of the secondary is difficult, and provides a large uncertainty to our derivation of the primary black hole mass. Even though there is no definitive way to measure the mass of the secondary, there are techniques to determine the mass ratio, $q$, for the system. Orosz et al. (1994) analyzed Balmer emission lines to find $K_{1}$ to use as an indicator of the motion of the primary. They then compared $\mathrm{K}_{1}$ to their $\mathrm{K}_{2}$ to obtain a $q=\mathrm{K}_{1} / \mathrm{K}_{2}=0.133 \pm 0.019$. Since their $\mathrm{K}_{1}$ probably arose in the accretion disk, it is only a limit to the true radial velocity of the primary. CMCMR calculated a system mass ratio of $q=0.128 \pm 0.04$ from combining their $v \sin i$ value for the secondary star 
with geometrical constraints. They were surprised to find that their mass ratio was close to the value found by Orosz et al. (1994). Fortunately, the orbital inclination of the system is relatively insensitive to the mass ratio (SNC). We will return to this point in section 3.6.

Interestingly enough, CMCMR did not detect a hot spot in their $\mathrm{H} \alpha$ Doppler map of GU Mus. This indicates that the $\mathrm{H} \alpha$ luminosity of the hot spot is smaller than, or equal to, that of the accretion disk. CMCMR did detect some emission from the accretion disk; however, and suggest that, "Perhaps the mass transfer rate in N. Mus is not sufficient to supply the luminosity of the hot-spot."

\section{2. $\quad$ Model Setup}

To model the infrared light curves, we have used WD98, the newest version of the WilsonDevinney light curve program (J. Kallrath 1999, private communication; R. E. Wilson 1999, private communication). WD98 is an enhanced version of WD95 (Kallrath et al. 1998), updated with new features such as the addition of semi-transparent circumstellar clouds, a simple spectral line profile capability for fast-rotating stars, an option to work with either observed times or phases, and the conversion of all the variables to double precision. Some of the relevant features of WD98 include: Kurucz atmosphere models for numerous wavelengths, a choice of three different limb darkening laws which are sensitive to changes in gravity, proximity and eclipse effects, the option for hot or cold stellar spots, and several different modes of operation for various system geometries. Descriptions of the earlier versions of the program can be found in papers by Wilson and Devinney (1971) and by Wilson $(1979,1990,1993)$. A recent application of WD95 can be found in Milone et al. (2000).

Briefly, WD98 works as follows. It takes the photospheres of the stars and divides them up into a multitude of surface elements. The amount of light coming from each element is calculated based on the binary system input parameters. All of these surface elements are then summed together, corrected for the line-of-sight geometry, to create the final light curve.

There are a number of input parameters needed to generate a model light curve for an SXT such as GU Mus. We discuss each of the most important of these parameters in the following subsections. We have made use of the best available system parameters. We list the most important wavelengthindependent input values to WD98 in Table 2, and the wavelength-dependent parameters in Table 3. Units are shown where appropriate. We ran WD98 in a mode set up to produce a model for a semi-detached binary with the secondary star automatically filling its Roche lobe (Mode $=5$ ), and

the primary having such a large gravitational potential, that it essentially has a radius of zero. We now discuss some of the input parameters in detail. 


\subsection{Limb Darkening}

The most important parameter that affects both the shape and the amplitude of the ellipsoidal variations is limb darkening. WD98 allows you to choose from several different forms of limb darkening: linear, logarithmic, or square-root. The linear law,

$$
I_{\lambda}(\mu)=I(1)\left(1-x_{\lambda}(1-\mu)\right),
$$

was first investigated by Milne in 1921. In this equation, $I_{\lambda}$ is the beam intensity at wavelength $\lambda$, $\mu$ is the cosine of the angle between the atmosphere normal and the beam direction, and $x_{\lambda}$ is the limb darkening coefficient. As an alternative to this, Klinglesmith \& Sobieski (1970) proposed the logarithmic law,

$$
\left.I_{\lambda}(\mu)=I(1)\left(1-x_{\lambda}(1-\mu)-y_{\lambda} \mu \ln (\mu)\right)\right),
$$

where $y_{\lambda}$ is the non-linear limb-darkening coefficient. Diáz-Cordovés \& Gimènez (1992) introduced the square-root law,

$$
I_{\lambda}(\mu)=I(1)\left(1-x_{\lambda}(1-\mu)-y_{\lambda}(1-\sqrt{\mu})\right) .
$$

When compared to ATLAS atmosphere models, the logarithmic law appears to fit UV models the best, while the square-root law appears better at infrared wavelengths (Van Hamme 1993). Models run by Claret (1998) for very low mass, solar metallicity stars $\left(2000 \mathrm{~K} \leq \mathrm{T}_{\text {eff }} \leq 4000 \mathrm{~K}\right)$ indicate that the square-root law best describes the intensity distribution in the infrared. We ran test models of stars with equal temperature and gravity, and found that the logarithmic and square-root laws produced nearly indistinguishable light curves. For the final models presented here, the square-root limb darkening law was adopted.

As shown by Alencar \& Vaz (1999) the limb darkening coefficients of stars in close binaries can be effected by irradiation. Even though there is no light coming from the primary object in an SXT, irradiation by the accretion disk and hot spot might exist. While CMCMR did not detect a hot spot in the GU Mus system, they did see a very strong signature from the secondary star. They attribute this to a very low mass transfer rate. They also estimated that the secondary star contributes $85-88 \%$ of the system flux in the $R$ band. Since the K4V secondary will dominate the system flux to a greater degree in the infrared, we have used the normal, non-irradiated, limb darkening coefficients. We present additional evidence for this in section 3.7.

\subsection{Gravity Darkening}

The second most important static parameter that affects the amplitude of the ellipsoidal variations is gravity darkening. Gravity darkening (a.k.a. brightening) deals with the localized temperature of a star's surface. The functional form for gravity darkening, $\mathrm{T}_{\text {eff }} \propto \mathrm{g}^{\beta}$, is wavelength independent, and is not strongly affected by changes in either the mixing length or the composition

of the star. The amount of gravity darkening depends on how energy is transported through the 
star, and thus is correlated with the mass of the star. For low mass, convective stars, $\beta \approx 0.32$, and for stars with radiative envelopes, $\beta \sim 1$ (Lucy 1967). For the cool secondary star used in our models, we have used a value of $\beta=0.38$ according to Claret (2000). He has calculated gravity darkening exponents for stars of mass $0.08 \mathrm{M}_{\odot}$ to $40 \mathrm{M}_{\odot}$, and has also found that a more accurate method of calculating gravity darkening exponents should include the shape of the star. Until that is done, however, we will use the gravity darkening exponent found by Claret (2000) for a $\mathrm{K} 4 \mathrm{~V}$ secondary with $\mathrm{T}_{\text {eff }}=4,500 \mathrm{~K}$. This gravity darkening exponent changes very little over the estimated spectral type range for the secondary star in GU Mus (K3V - K5V).

\subsection{Other input parameters}

There are a variety of other input parameters with less freedom in their selection. For example, we must choose the values for the temperature and monochromatic luminosities of the secondary star. For our models, we used a $\mathrm{K} 4 \mathrm{~V}$ secondary temperature of $\mathrm{T}_{\text {eff }}=4,500 \mathrm{~K}$, a $J$ band luminosity of $\mathrm{L}_{J}=0.311 \mathrm{~L}_{\odot}$, and a $K_{s}$ band luminosity of $\mathrm{L}_{K s}=0.421 \mathrm{~L}_{\odot}$.

The atmospheres of cool stars are fairly complicated and the details of their spectral energy distributions and any changes in such, as a function of temperature, make for complex modeling (cf., Allard et al. 1997). Stellar atmosphere codes have to take into account numerous atomic and molecular absorption features which affect the limb darkening coefficients. In order to accurately model the limb darkening effects of the secondary star in GU Mus, we have used the Kurucz atmosphere models incorporated into WD98.

Irradiation of the secondary star atmosphere in close binaries such as GU Mus can be very important. Harlaftis \& Filippenko (2000) used doppler tomography to show evidence of a hot spot in QZ Vul (=GS 2000+25) where the accretion stream meets the accretion disk. WD98 can calculate reflection/re-radiation of this type of irradiation based on the bolometric albedos of the two stars. The expected value for radiative envelopes is unity, while the bolometric albedo for the convective secondary star is expected to lie somewhere between 0.5 and 1 , based on models run by Nordlund and Vaz (Nordlund \& Vaz 1990; Vaz \& Nordlund 1985). This value is dependent on the amount of convection in the star: the smaller the mixing length parameter, $\alpha=l / H_{p}$, the closer it is to a radiative atmosphere, and the higher the bolometric albedo. In the case of GU Mus, we used a bolometric albedo of 0.676 based on the average of the albedos given in Table 3 of Nordlund \& Vaz (1990); however, this parameter was irrelevant since there is little evidence for other significant sources of luminosity in the system.

Our GU Mus $J$ and $K_{s}$ band observations and their corresponding WD98 models for five different values of the orbital inclination angle $\left(50^{\circ}, 53^{\circ}, 55^{\circ}, 57^{\circ}\right.$, and $\left.60^{\circ}\right)$, are presented in Figures 3 and 4, respectively. The points represent the data while the curves represent the models.

We present five different inclinations to demonstrate how a change in inclination angle affects the resulting light curve in these two band passes. For the models shown in Figs $3 \& 4$, a $\chi^{2}$ test gives 
$i=55^{\circ}$ as the best fit to the data. For a fixed set of system parameters, we have found that $\chi^{2}$ tests can distinguish between orbital inclinations of $\pm 1^{\circ}$.

\subsection{Sensitivity of the light curve shape to input parameter variation}

We have just shown how we arrived at our input parameters. Many of the parameters discussed have simply been set to values consistent with the system parameters of GU Mus. Several of these input parameters, however, are either derived from observations of GU Mus $\left(\mathrm{T}_{e f f}\right)$, or are unknown (e.g., the orbital inclination or mass ratio). In order to quantify the sensitivity of the models to the variations of these parameters, we ran models with inputs covering a wide range of parameter space.

Varying $\mathrm{T}_{\text {eff }}$ from 4,400 K (K5V) to 4,800 K (K3V), the observed spectral type range of the secondary star, did not produce significant differences in the light curves. Thus for this entire range of temperatures, with $q=0.10$, the best fit orbital inclination angle is $i=53^{\circ}$. Since we did not know the exact mass of either the primary or secondary star in GU Mus, the input values of $q$

had to be estimated from previous results. To test the sensitivity of the light curve shape to the mass ratio, we ran models with $q$ 's covering a very large range: $0.08 \leq q \leq 0.13 . \chi^{2}$ minimization tests were performed on all of these models to determine the best fit orbital inclination angle. For models with a mass ratio of $q=0.13$, we found a best fit inclination angle of $i=55^{\circ}$. For $q \leq 0.1$, we found that $i=53^{\circ}$ gave the smallest $\chi^{2}$ value. Thus, our resulting inclination angle will not be very sensitive to $q$.

\subsection{Sensitivity of the light curve shape to other sources of luminosity in the system}

In the previous sections, we have only considered the light from the secondary star. If there are other sources of luminosity in the system, they can affect our result for the orbital inclination angle. For example, excess light from a constant source other than the secondary star will dilute the ellipsoidal variations. Before we proceed any further, we need to investigate the possibility that other sources of luminosity exist in this system.

As discussed above, CMCMR did not detect a hot spot. They did, however, see evidence for emission from a disk. If the accretion disk in GU Mus were to contribute infrared light to the system, the true orbital inclination angle would be larger than that derived due to the dilution of the light from the secondary star. CMCMR suggested that there was a small (12-15\%) non-stellar contribution to the GU Mus flux in the $R$ band. If we consider a worst case scenario where the contamination at $J$ is the same as that observed at $R(15 \%)$, the derived orbital inclination angle is increased by four degrees. Due to the relatively flat nature of model accretion disk spectra (e.g, $f_{\lambda} \propto \lambda^{-2}$; Oke 1977), the contamination will almost certainly be less at $J$ than at $R$. Assuming this $15 \%$ level of contamination and taking into account the range of mass ratios given in the previous 
section, we obtain an orbital inclination angle of $54_{-1.5}^{o+4}$.

We now present evidence which suggests that the level of contamination in the GU Mus system is significantly smaller than stated above. All of the previous optical studies clearly show unequal maxima in the observed light curves of GU Mus (OBMR, for example). This implies that one side of the star is heated by the accretion disk, and that contamination is an important factor in the modeling of the light curve. The observed infrared light curves presented here have equal maxima, and thus we do not see evidence for accretion disk irradiation of the secondary star. This suggests a minimal level of heating and/or reflection in the GU Mus system.

We can also investigate the level of phase-dependent contamination in GU Mus by noting any changes in the apparent color of the system throughout its orbit. The infrared color at maximum phase will differ from that at minimum phase if either a hotter or cooler source of luminosity exists within the system. As can be seen in Figures $3 \& 4$, the $J-K$ colors agree at both maximum and minimum phase, thus again ruling out a significant phase-dependent contamination.

Given that the expected spectral energy distribution of an SXT at minimum light would be more strongly contaminated in the optical portion of the spectrum, we can further examine the level of contamination by looking at the optical colors of GU Mus. In Table 1, we present the colors of GU Mus and compare the averages to the colors of a $\mathrm{K} 4$ dwarf and giant (reddened by $\mathrm{A}_{\mathrm{V}}=$ $0.9 \mathrm{mag}$ ). The colors of GU Mus are intermediate to those of the K4 giant and dwarf colors. Thus, there is very little evidence for significant blue sources of light in the system. The $V-R$ color is slightly redder $(\sim 10 \%)$ than the dwarf or giant, but can be explained by emission from $\mathrm{H} \alpha$.

Thus, we do not see significant evidence for emission from the accretion disk itself, or its irradiation of the secondary star. But even in the most conservative scenario, the effect on our derived orbital inclination angle is $\leq 4^{\circ}$.

\section{Results}

We have explored a wide range of parameter space for GU Mus, and have found that the model light curves are relatively insensitive to the input parameters, except for the inclination angle. Even changing the mass ratio, $q$, by a very large amount (i.e., changing the secondary star mass from $0.56 \mathrm{M}_{\odot}$ to $0.90 \mathrm{M}_{\odot}$ for a primary mass of $6.95 \mathrm{M}_{\odot}$ ), changes the derived orbital inclination angle of the system by only two degrees $\left( \pm 1^{o}\right)$. We have considered other sources of luminosity in the system and even under the most conservative scenario, the derived orbital inclination angle is

$54_{-1.5}^{o+4}$. We believe, however, that the infrared light curve is not seriously affected by any such contamination. Within the ability of WD98 to realistically model the light curves of SXTs, the derived orbital inclination of $G U$ Mus is $54 \pm 1.5^{\circ}$. Our derived orbital inclination angle agrees with SNC's inclination determined from their $H$ band light curve of GU Mus.

With the orbital inclination angle determined, we can estimate the mass of the primary. To 
calculate the mass of the primary object in GU Mus, we need to know three things: the orbital inclination, the radial velocity of the secondary star, and the mass of the secondary. The radial velocity of the secondary star is $\mathrm{K}_{2}=406 \pm 7 \mathrm{~km} \mathrm{~s}^{-1}$ (OBMR). From the observed range in spectral types, the main sequence mass of the secondary star is $0.75 \pm 0.05 \mathrm{M}_{\odot}$ (Baraffe et al. 1998). (A large uncertainty in finding the mass of the primary remains that associated with the mass of the secondary star. As noted by both CMCMR and OBMR, the secondary star in this system may be slightly evolved. Even though the mass of the secondary in GU Mus did not have a significant effect on our orbital inclination angle determination, it plays a large roll in finding the mass of the primary.) Using these values, we calculate a black hole mass of $6.95 \pm 0.6 \mathrm{M}_{\odot}$. This mass is within the range reported by both CMCMR and SNC.

For an average primary mass of $6.95 \mathrm{M}_{\odot}$, and an average secondary mass of $0.75 \mathrm{M}_{\odot}$, we obtain a $q=0.108$. Using this mass ratio and a semi-major axis of $4.6 \mathrm{R}_{\odot}$, we find the resulting Roche lobe radius of the secondary star in GU Mus to be $0.97 \mathrm{R}_{\odot}$. Note that a main sequence K4V would have a radius of $0.73 \mathrm{R}_{\odot}$, thus filling $\sim 75 \%$ of the derived Roche lobe radius. Interestingly, both the derived radius and optical colors are consistent with the secondary star being a subgiant. Using $\mathrm{R}=0.97 \mathrm{R}_{\odot}$ and $\mathrm{T}_{\text {eff }}=4,500 \mathrm{~K}$ (appropriate for a $\mathrm{K} 4 \mathrm{~V}$ ), we derive a bolometric luminosity of $\mathrm{L}_{B o l}=0.34 \mathrm{~L}_{\odot}$. Using our infrared photometry, corrected for reddening, we determine a distance to GU Mus of $5.1 \mathrm{kpc}$.

The development of a consistent predictive theory of accretion flows around black holes is one of the main goals of high-energy astrophysics. The origin of the outbursts of SXTs can be explained by the accretion disk limit cycle mechanism that was developed to describe the outbursts of cataclysmic variables (Cannizzo 1998). Modeling of the outbursts of SXTs, from the x-ray through optical, has been attempted by a number of researchers. The consensus appears to be that the optical and soft x-ray luminosities are generated within an accretion disk. The hard x-ray spectra that develop later in the outburst have been explained as advection dominated accretion onto the black hole. Depending on the model chosen to explain the multi-wavelength luminosities, the inclination angle can play an important role in the appearance of the outburst.

For example, Esin, McClintock, and Narayan (1997) calculated theoretical x-ray models to fit to various phases that GU Mus exhibited during its outburst. They combined a normal accretion disk with an advection dominated accretion flow (ADAF). In their models, they input a black hole mass of $6 \mathrm{M}_{\odot}$, an inclination angle of $i=60^{\circ}$, and a distance of $5 \mathrm{kpc}$, taken from OBMR, CMCMR, and SNC. They found that the during the outburst of GU Mus, both the accretion disk and ADAF remained optically thin. If so, the inclination angle does not play a serious role in what is observed. But as Esin et al. (2000) note, even if the ADAF is optically thin, the emission will preferentially escape along the polar axes, and thus the inclination angle may affect the observed x-ray flux. In contrast, Misra (1999), uses a similar model (with identical values of $\mathrm{M}_{1}$, $i$, and distance), but one where both the ADAF and the accretion disk are optically thick. If this model is correct, then the inclination angle plays an important role in what is observed. New models that are constructed to explain the outburst of GU Mus should take into account our new results for the mass of the black 
hole, the orbital inclination angle and the distance.

\section{Conclusions}

We have obtained new $J$ and $K_{s}$ band light curves covering a complete orbit for GU Mus. We have used WD98 to model the resulting light curves, and obtained an orbital inclination of $54 \pm 1.5^{\circ}$. This leads to a primary mass estimate of $6.95 \pm 0.6 \mathrm{M}_{\odot}$. In quiescence, GU Mus is a relatively simple system to model: There is no hot spot, and very little accretion to contaminate its infrared light curves. There is also a consensus on the orbital inclination angle for GU Mus, unlike the other SXTs. Thus, it provides the opportunity to test our modeling procedure before attempting more complex systems.

Before we can fully understand the outbursts of SXTs, we need to determine values for the most important systemic parameters. Unfortunately, it is much harder to determine system parameters for non-eclipsing binaries, than for their eclipsing counterparts. The only way to determine the orbital inclination in a non-eclipsing system is to model its ellipsoidal variations. WD98 allows us to model these variations using the most up-to-date software package available. By modeling infrared data, where the contamination from non-stellar sources is minimized, we have a robust technique to determine important orbital parameters.

DMG would like to thank Josef Kallrath and Bob Wilson for the use of WD98, as well as their help in running and understanding the program. This research was supported by a Grant-in-Aid of Research from the National Academy of Sciences, through Sigma Xi, The Scientific Research Society. DMG holds an American fellowship from the American Association of University Women Educational Foundation. TEH wishes to acknowledge support from the Small Research Grants program of the American Astronomical Society, the New Mexico Space Grant Consortium, and the New Mexico State University Arts and Sciences Minigrant program. We would also like to thank the anonymous referee for some useful comments which improved this paper. 


\section{REFERENCES}

Allard, F., Huaschildt, P., Alexander, D., \& Starrfield, S. 1997, ARA\&A, 35, 137

Alencar, S.H.P., \& Vaz, L.P.R. 1999, A\&A Supp., 135, 555

Antokhina, Eh. A., \& Cherepashchuk, A. M. 1993, Pis'ma v Astronomicheskij Zhurnal, 19, \#6, 500

Baraffe, I., Chabrier, G., Allard, F., \& Hauschildt, P.H. A\&A, 1998, 337, 403

Bessell, M.S. 1991, AJ, 101, 662

Cannizzo, J. K. 1998, in ASP Conf. Ser., 137, ed. S. Howell, E. Kuulkers, \& C. Woodward (San Francisco: ASP), 308

Casares, J., Martín, E.L., Charles, P.A., Molaro, P., \& Rebolo, R. 1997, NewA, 1, 299 (CMCMR)

Claret, A. 1998, A\&A, 335, 647

Claret, A. 2000, A\&A, 359, 289

Della Valle, M., Masetti, N., \& Bianchini, A. 1998, A\&A, 329, 606

Diáz-Cordovés, J., \& Giménez, A. 1992, A\&A, 259, 227

Esin, A. A., Kuulkers, E., McClintock, J. E., \& Narayan, R. 2000, ApJ, 532, 1069

Esin, A. A., McClintock, J. E., \& Narayan, R. 1997, ApJ, 489, 865

Harlaftis, E.T., \& Filippenko, A.V. 2000, in Proc. SPIE, 4005, Discoveries and Research Prospects from 8 to 10 - Meter Class Telescopes, ed. J. Bergeron, (New York: Springer), 232

Hua, X., \& Lingenfelter, R.E. 1993, ApJ, 416, L17

Kallrath, J., Milone, E.F. 1999, Eclipsing Binary Stars: Modeling and Analysis (New York: Springer)

Kallrath, J., Milone, E.F., Terrell, D., \& Young, A.T. 1998, ApJ, 508, 308

King, N. L., Harrison, T. E., \& McNamara, B. J. 1996, AJ, 111, 1675

Klinglesmith, D.A., \& Sobieski, S. 1970, AJ, 75, 175

Kurucz, R.L., 1993, in Light Curve Modeling of Eclipsing Binary Stars, ed. E.F. Milone (New York: Springer), 93

Lucy, L.B. 1967, Zeitschr für Astrophys, 65, 89

Lund, H., Brandt, S. 1991, IAU Circ. 5161

Makino, F. et al. 1991, IAU Circ. 5161

Milne, E.A. 1921, MNRAS, 81, 361

Milone, E.F., Schiller, S.J., Munari, U., \& Kallrath, J. 2000, ApJ, 199, 1405

Misra, R. 1999, ApJ, 512, 340 
Nordlund, A ., \& Vaz, L.P.R. 1990, A\&A, 228, 231

Oke, J.B. 1977, ApJ, 217, 182

Orosz, J.A., Bailyn, C.D., Remillard, R.A., McClintock, J.E., \& Foltz, C.B. 1994, ApJ, 436, 848

Orosz, J.A., Bailyn, C.D., McClintock, J.E., \& Remillard, R.A. 1996, ApJ, 468, 380 (OBMR)

Pogge, R.W., Martini, P., \& DePoy, D.L. 1999,

http://www.ctio.noao.edu/instruments/ir_instruments/osiris/manual/

Remillard, R.A., McClintock, J.E., \& Bailyn, C.D. 1992, ApJ, 399, L145

Remillard, R.A., Schachter, J.F., Silber, A.D., \& Slane, P. 1994, ApJ, 426, 288

Shahbaz, T., Naylor, T., \& Charles, P.A. 1997, MNRAS, 285, 607 (SNC)

van Hamme, W. 1993, AJ, 106, 2096

Vaz, L.P.R., \& Nordlund, A . 1985, A\&A, 147, 281

Wilson, R.E. 1979, ApJ, 234, 1054

Wilson, R.E. 1990, ApJ, 356, 613

Wilson, R.E. 1993, in ASP Conf. Ser. 38, New Frontiers in Binary Star Research, ed. K.C. Leung \& I.S. Nha (San Francisco: ASP), 91

Wilson, R.E. 1998, in Reference Manual to the Wilson-Devinney Program, Computing Binary Star Observables, Version 1998 (Gainsville, FL: Univ. Florida)

Wilson, R.E., \& Devinney, E.J. 1971, ApJ, 166, 605 


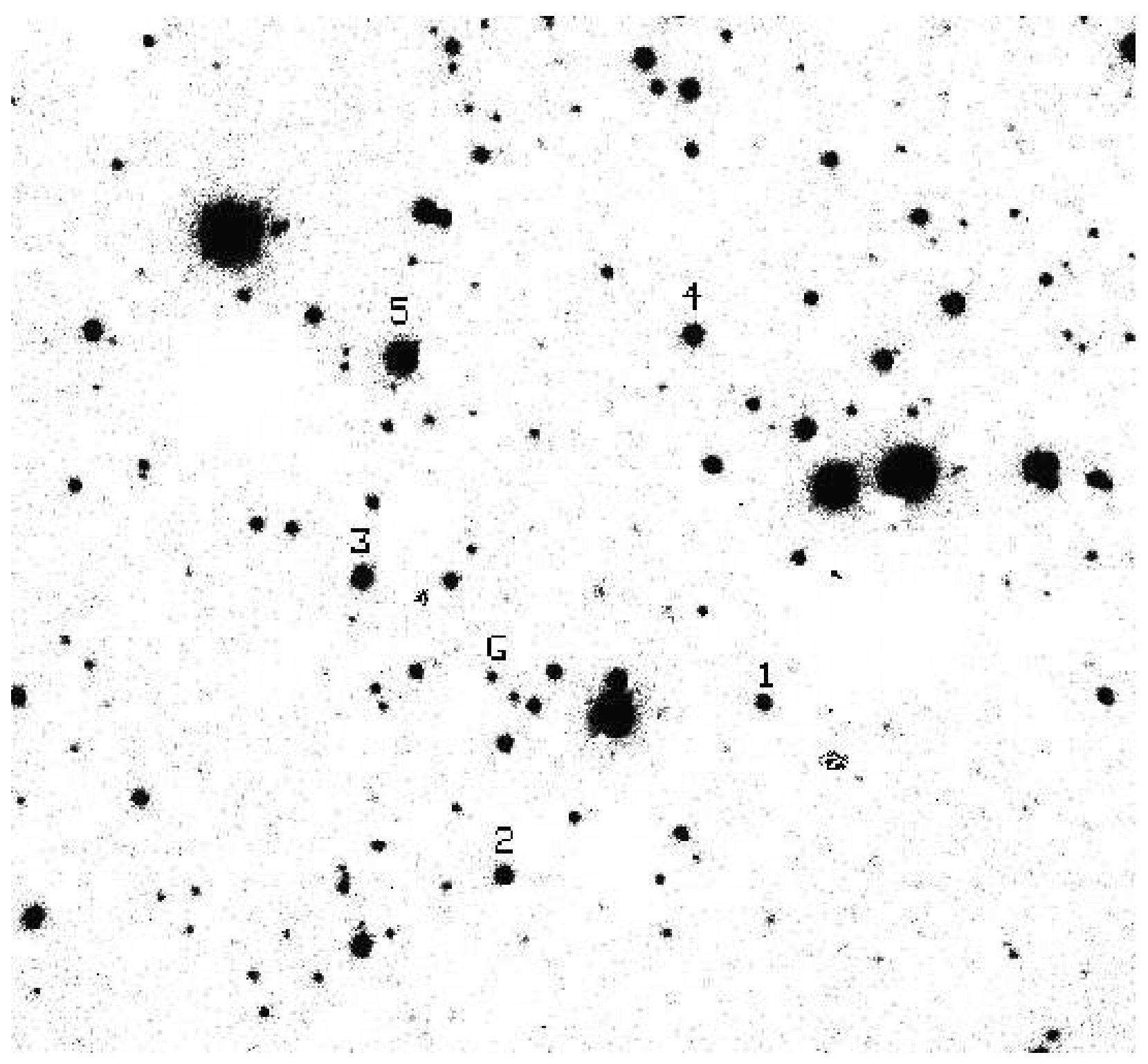

Fig. 1. - OSIRIS $J$ band image of GU Mus $\left(\alpha_{2000}=11: 26: 26.7, \delta_{2000}=-68: 40: 32.6\right)$ taken on February 20, 2000 with the Victor M. Blanco 4 meter telescope at Cerro Tololo Inter-American Observatory. The field is 1.5 ' x 1.5 ' with a scale of 0.16 " / pixel. The SXT is labeled with a "G". The five comparison stars used for the differential photometry are numbered. 

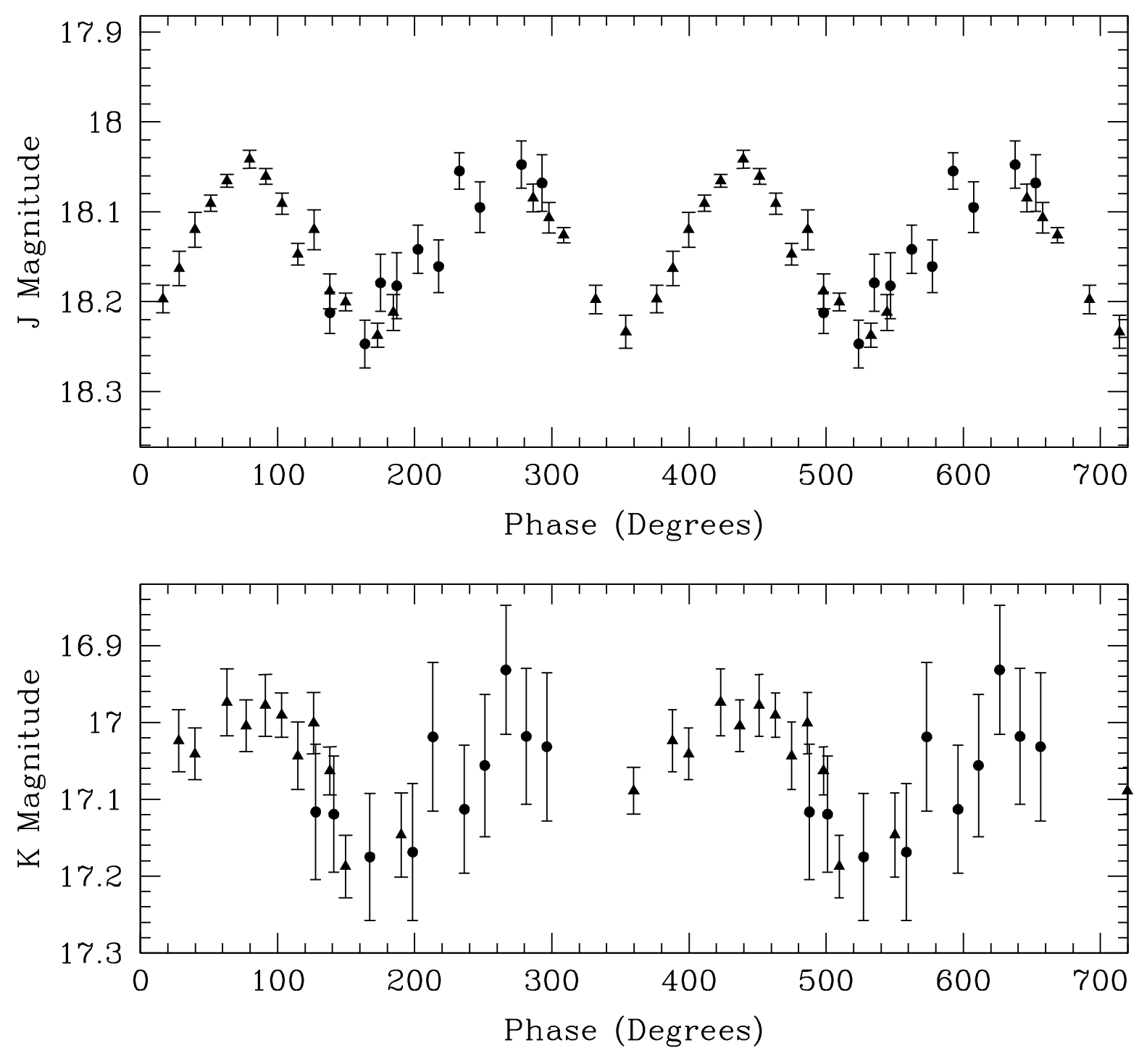

Fig. 2.- GU Mus $J$ band (top panel) and $K_{s}$ band (bottom panel) light curves. The data were obtained on February 20 (triangles) and February 21 (circles), with OSIRIS on the CTIO Victor M. Blanco 4-m telescope. The data are plotted over two phase cycles for clarity. Here and throughout this paper we phase our heliocentric corrected data to the Shahbaz et al. (1997) ephemeris. 


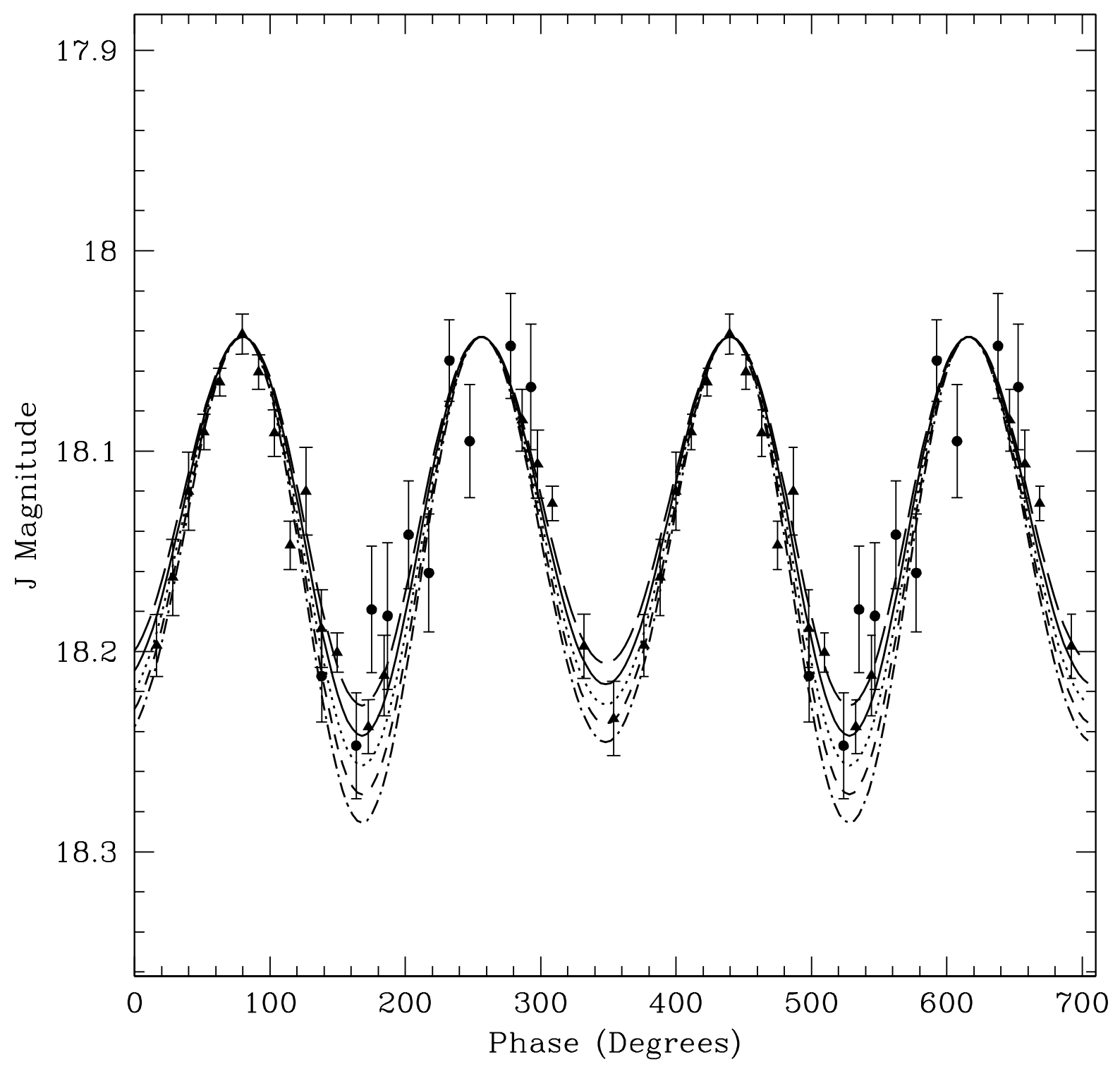

Fig. 3. $-J$ band light curve with five WD98 models. The data are the same as in Figure 2. The lines are WD98 models of differing orbital inclination angles: $50^{\circ}$ (long-dashed), $53^{\circ}$ (solid), $55^{\circ}$ (dotted), $57^{\circ}$ (short-dashed), and $60^{\circ}$ (dot dashed). With $q=0.13$, a $\chi^{2}$ minimization finds the best fit model to be $55^{\circ}$. 


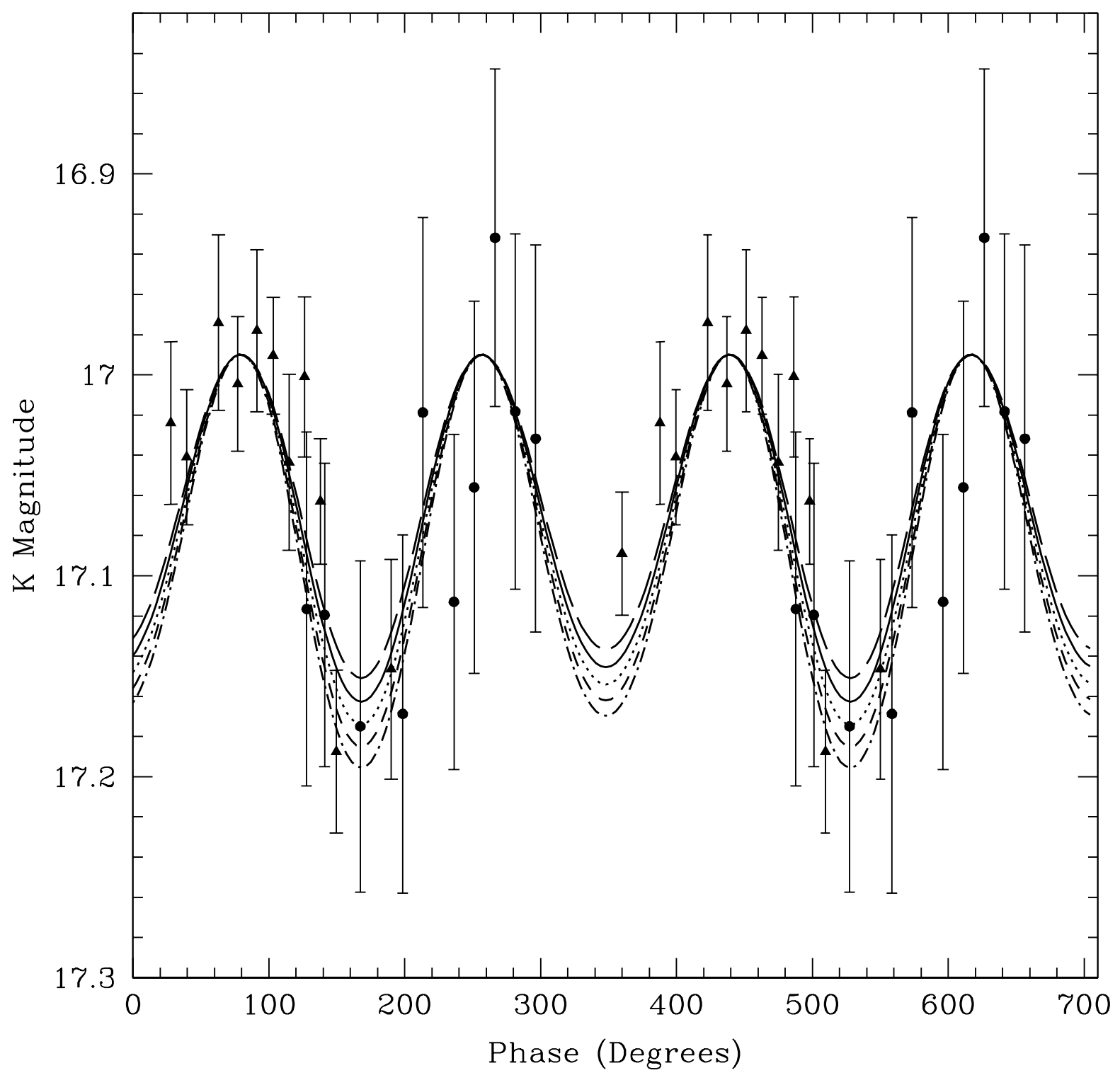

Fig. 4. $-K_{s}$ band light curve with the five WD98 models as in Figure 3. 
Table 1. Quiescent Infrared and Optical Colors of GU Mus

\begin{tabular}{|c|c|c|c|c|c|c|c|}
\hline Reference & $\mathrm{V}$ & $\mathrm{B}-\mathrm{V}$ & $\mathrm{V}-\mathrm{R}$ & $\mathrm{V}-\mathrm{I}$ & $\mathrm{J}$ & $\mathrm{J}-\mathrm{K}$ & Year $^{\mathrm{a}}$ \\
\hline 1 & $20.5 \pm 0.1$ & 1.60 & 1.00 & $\ldots$ & $\ldots$ & $\ldots$ & 1992 \\
\hline 2 & $20.66 \pm 0.03$ & $\ldots$ & 0.91 & 1.66 & $\ldots$ & $\ldots$ & 1992 \\
\hline 3 & $20.55 \pm 0.05$ & $\ldots$ & $\ldots$ & 1.53 & $\ldots$ & $\ldots$ & 1992 \\
\hline 4 & $20.35 \pm 0.03$ & $\ldots$ & $\ldots$ & 1.55 & $\ldots$ & $\ldots$ & 1993 \\
\hline 5 & $20.83 \pm 0.06$ & $\ldots$ & $0.90 \pm 0.07$ & $1.83 \pm 0.07$ & $18.14 \pm 0.02$ & $1.06 \pm 0.10$ & $2000 / 2001$ \\
\hline Mean Observed Color & & $1.60 \pm 0.06$ & $0.93 \pm 0.05$ & $1.64 \pm 0.14$ & & $1.06 \pm 0.10$ & \\
\hline $\mathrm{K} 4 \mathrm{~V}\left(\mathrm{~A}_{\mathrm{V}}=0.9 \mathrm{mag}\right)$ & & 1.36 & 0.77 & 1.53 & & 0.83 & \\
\hline $\operatorname{K} 4 \mathrm{III}\left(\mathrm{A}_{\mathrm{V}}=0.9 \mathrm{mag}\right)$ & & 1.70 & 0.88 & 1.87 & & 1.03 & \\
\hline
\end{tabular}

${ }^{a}$ Year the data were taken

References. - (1) Della Valle et al. 1998; (2) King et al. 1996; (3) Remillard et al. 1992; (4) OBMR; (5) This Paper 
Table 2. Wavelength Independent WD98 Input Parameters for GU Mus

\begin{tabular}{lc}
\hline \hline \multicolumn{1}{c}{ Parameter } & Value \\
\hline Orbital Period (days) & 0.432604 \\
Ephemeris $^{\mathrm{a}}$ (HJD phase 0.0) & 2448812.669 \\
Semi-Major Axis $\left(\mathrm{R}_{\odot}\right.$ ) & 4.6 \\
Orbital Eccentricity & 0.0 \\
Temperature of K4V Secondary $(\mathrm{K})$ & 4,500 \\
Mass Ratio $\left(\mathrm{M}_{2} / \mathrm{M}_{1}\right)$ & 0.108 \\
Atmosphere Model & Kurucz \\
Limb Darkening Law & Square-root \\
Secondary Star Gravity Darkening Exponent & $\beta=0.38$ \\
Secondary Star Bolometric Albedo & 0.676 \\
\hline
\end{tabular}

${ }^{a}$ From Shahbaz, Naylor, and Charles (1997) 
Table 3. Wavelength Dependent WD98 Input Parameters for GU Mus

\begin{tabular}{ccc}
\hline \hline Parameter & $\mathrm{J}$ & $\mathrm{K}_{s}$ \\
\hline Secondary Star Monochromatic Luminosity $\left(\mathrm{L}_{\odot}\right)$ & 0.311 & 0.421 \\
Secondary Star Square-root Limb Darkening Coefficients: & & \\
$\mathrm{x}_{\lambda}$ & 0.110 & -0.116 \\
$\mathrm{y}_{\lambda}$ & 0.531 & 0.724 \\
\hline
\end{tabular}

